OPEN ACCESS

Edited by:

Ali Mentes,

Marmara University, Turkey

Reviewed by:

Ece Eden,

Ege University, Turkey

Jilen Patel,

University of Western

Australia, Australia

*Correspondence:

Andrea Ferreira Zandona

Andrea.Zandona@tufts.edu

Specialty section: This article was submitted to

Pediatric Dentistry,

a section of the journal

Frontiers in Dental Medicine

Received: 08 November 2021 Accepted: 29 November 2021

Published: 17 December 2021

Citation:

Prakash M, Kang YH, Jain S and

Zandona AF (2021) In-vitro

Assessment of Silver Diamine Fluoride

Effect on Natural Carious Dentin

Microhardness.

Front. Dent. Med. 2:811308.

doi: 10.3389/fdmed.2021.811308

\section{In-vitro Assessment of Silver Diamine Fluoride Effect on Natural Carious Dentin Microhardness}

\author{
Meghna Prakash ${ }^{1}$, Yoon H. Kang ${ }^{1}$, Shruti Jain ${ }^{2}$ and Andrea Ferreira Zandona ${ }^{\text {* }}$ \\ ${ }^{1}$ Department of Comprehensive Care, Tufts University School of Dental Medicine, Boston, MA, United States, ${ }^{2}$ Department \\ of Biostatistics and Experimental Design, Tufts University School of Dental Medicine, Boston, MA, United States
}

This study aimed to assess the effect of silver diamine fluoride (SDF) on natural carious dentin microhardness and the correlation between tactile sensation, fluorescence, and microhardness on carious dentin. Permanent carious teeth scored ICDAS 4-6 were longitudinally sectioned into tooth slabs exposing carious dentin on one side and sound dentin on the other. Both sides were assessed for tactile sensation (soft/leathery/firm), fluorescence (red/pink/no fluorescence) with FACE technology (Sirolnspect ${ }^{\circledR}$, Dentsply Sirona, USA), and Vickers's microhardness (VMH). Samples were randomized into 3 groups based on SDF protocol $(n=30)$ : Control/Group A- No SDF treatment; Group B- 38\% SDF (Advantage Arrest ${ }^{\mathrm{TM}}$, Elevate Oral Care ${ }^{\circledR}$, USA); Group C- SDF with potassium iodide/KI (Riva Star, SDI, Australia). After SDF application, all samples were stored for 1 week under $100 \%$ humidity at $37^{\circ} \mathrm{C}$ and re-assessed for tactile sensation, fluorescence, and microhardness. Change in microhardness ( $\triangle \mathrm{VMH}$; mean $\pm \mathrm{SD}$ ), tactile sensation $(\Delta T)$, and fluorescence $(\Delta F)$ were calculated using the difference between pre-SDF and post-SDF values. Mixed ANOVA analysis showed that $\triangle \mathrm{VMH}$ of carious and sound surfaces were statistically significantly higher for each of the experimental groups (Group B-sound: $20.22 \pm 11.98 \mathrm{HV}$, carious: $19.76 \pm 9.35 \mathrm{HV}$; Group C-sound: $14.26 \pm 10.11 \mathrm{HV}$, carious: $22.51 \pm 7.67 \mathrm{HV}$ ) than the control group (Group A-sound: $7.34 \pm 8.28 \mathrm{HV}$, carious: $0.69 \pm 3.53 \mathrm{HV})(p<0.0001)$. There was no statistically significant difference between the experimental groups themselves for carious surfaces ( $p=0.146)$. On sound surfaces, Group B showed a statistically significantly higher $\Delta \mathrm{VMH}$ than Group C ( $p=0.026)$. There was no statistically significant interaction between type of surface and $\triangle \mathrm{VMH}$ in Group $\mathrm{B}(p=0.809)$. In Group $\mathrm{C}$, sound surfaces showed a statistically significantly lower $\Delta \mathrm{VMH}$ than carious surfaces $(p<0.0001)$. Spearman rank-order correlation showed a statistically significant negative correlation between $\Delta \mathrm{VMH}$ and $\Delta \mathrm{T}(r s=-0.588, p<0.0001)$ and between $\Delta \mathrm{VMH}$ and $\Delta \mathrm{F}$ ( $r s=-0.269, p=0.01)$. There was a statistically significant positive correlation between $\Delta \mathrm{T}$ and $\Delta \mathrm{F}$ ( $r \mathrm{~s}=0.226, p=0.032)$. In conclusion, the microhardness of SDF-treated dentin surfaces increased as compared to non-SDF-treated surfaces. SDF (Advantage Arrest ${ }^{\mathrm{TM}}$ ) increased microhardness of carious and sound dentin to a similar extent whereas, SDF/KI (Riva Star) increased microhardness of carious dentin more than sound dentin. An increase in microhardness was correlated with a firmer tactile sensation.

Keywords: silver diamine fluoride, caries, microhardness, dentin remineralization, fluoride 


\section{INTRODUCTION}

Evolving research in the field of cariology has brought about a paradigm shift in the understanding of the caries process and its management. With respect to caries management, preserving natural tooth integrity by proper prevention, risk assessment, accurate diagnosis, and timely treatment procedures that do not compromise sound tooth structure is essential (1).

Traditional clinical protocols for the management of carious lesions involved complete caries removal whereby total excavation of presumably infected and affected dentin was performed until clinically sound and firm/hard is reached followed by the placement of a restoration (2). However, with the subjective nature of caries excavation, these highly invasive procedures pose a risk of pulp exposures and compromised tooth integrity (3). Teeth with failed restorative treatment become candidates for complex and expensive endodontic procedures, and go further down the restorative "death spiral" (4). In clinical situations where deep carious lesions approximate a vital pulp, the decision to excavate or not is crucial. Compounding evidence indicates that removal of all soft, infected dentin in cavitated lesions may not be necessary; a well-sealed restoration over a cavitated lesion could arrest the lesion, reducing bacterial count over time $(2,5)$. In recent years, selective caries excavation limited to the outer soft and friable carious dentin has gained popularity as a conservative and cost-effective alternative to complete caries removal with potential for reducing the risk of pulp exposures (6-8). In a worldwide consensus statement by the European Organization for Caries Research (ORCA) and the European Federation of Conservative Dentistry (EFCD) on recommendations for caries interventions in children, it was recommended that cavitated deep carious lesions in permanent posterior teeth may be treated by selective carious tissue removal and a well-sealed restoration (9). Yet, selective caries removal continues to have a low acceptance rate among many clinicians due to concerns with leaving bacteria in the cavity (10-12).

Silver diamine fluoride (SDF) was approved by the US Food and Drug Administration in 2014 as a desensitizing agent, and has shown promise as an effective, off-label caries control agent. It serves as a minimally invasive treatment option for caries by virtue of its caries-arresting effect, thereby limiting the need for operative intervention. Silver diamine fluoride consists of $25 \%$ silver, $5 \%$ fluoride, and $8 \%$ ammonia. It has antimicrobial (due to the action of silver), remineralizing (due to fluoride) and biofilm inhibiting functions $(13,14)$. Aside from the unaesthetic dark stain it produces, SDF has minimal documented adverse effects limited to rare transient mucosal lesions (15). Black staining is the result of the formation of a silver phosphate layer and a silver sulfide precipitate on carious dentin and can affect the acceptance of SDFs among patients (16). Reportedly, dark staining can be reduced by the use of a saturated solution of potassium iodide (KI), which reacts with the remaining free silver ions to form silver iodide, giving a white creamy reaction product (17).

Affected dentin has been theorized to have the potential to remineralize as it structurally maintains enough collagen integrity (18). Arresting caries entails inactivating the lesion by removing or altering the biofilm, halting its progression and remineralizing demineralized dentin. It has been widely acknowledged that SDF has caries-arresting properties and can initiate a reparative process through remineralization. However, there is limited knowledge on whether SDF's remineralizing ability can bring about mineral gain in dentinal carious lesions corresponding to the mineral content of sound dentin. Measuring dentin hardness can be considered as an acceptable and indirect method of estimating the changes in mineral content of dentin (19). Determining the effect of SDF on the hardness of dentin, the primary aim of this study, becomes useful in such scenarios, as there could be a possibility of remineralizing and hardening caries-affected dentin tissues to the extent of a sound dentin surface. In turn, any cariostatic and hardening effect of SDF when combined with the minimally invasive nature of selective caries excavation can further encourage and aid clinicians to conservatively manage deep caries in daily clinical practice.

Clinically, arresting of the caries process after SDF treatment is commonly verified by assessing tactile hardness of the lesion (2022). According to the American Dental Association's evidencebased clinical practice guidelines on non-restorative treatments for carious lesions, when a carious lesion turns firm/hard to clinical tactile probing, it is considered arrested (23). However, the nature of these chair side examinations remains highly subjective and requires calibration among examiners to provide a reliable measure of mineral gain/loss. Conversely, conventional microhardness tests routinely used in in-vitro studies are laboratory techniques that cannot be implemented chair side. In a clinical setting, it is impractical to use these conventional systems to determine outcome of SDF-treated dentin. A secondary aim of this study was to assess the correlation of microhardness to clinically viable parameters like fluorescence or tactile sensations of carious dentin. Understanding this correlation is advantageous for clinicians to implement a chair side assessment of SDF treatment outcomes with regards to improving dentin hardness.

\section{MATERIALS AND METHODS}

Extracted and de-identified posterior permanent human teeth were selected from available teeth collected within the last 6 months in the Cariology lab from which 90 sliced samples were obtained $(N=90)$ under an IRB-exempt protocol approved by the Dental Research Administration at Tuft University School of Dental Medicine. Included teeth had coronal caries scored 46 in the International Caries Detection and Assessment System (ICDAS) in order to acquire samples with lesions on coronal dentin. Any sound teeth, anterior teeth, fluorosed teeth, restored teeth or teeth with developmental defects on dentin or enamel were excluded from the study. The teeth were previously stored in $0.2 \%$ thymol solution at $4^{\circ} \mathrm{C}$. Prior to processing, the selected teeth were rinsed with deionized water to remove any debris.

\section{Sample Preparation}

For the purpose of this study, the stored carious teeth were mounted in self-curing resin $\left(\right.$ Caulk $^{\circledR}$ Orthodontic Resin, DENTSPLY) and sectioned into two halves along the root length through the center of the lesion under continuous water-cooling 
using an automatic grinding machine (Isomet ${ }^{\mathrm{TM}}$ 100, Buehler, Lake Bluff, IL, USA). Each half was further sectioned through sound dentin on the other side of the caries-sound dentin interface to obtain a longitudinal slice. As a result, each tooth was made into two sample slices, each containing carious dentin on one surface and sound dentin on the other surface. A schematic diagram of the sectioning planes used for sample preparation is shown in Figure 1. Sliced samples $(N=90)$ were wetground polished (EcoMet ${ }^{\mathrm{TM}}$ 250, Buehler, Lake Bluff, IL, USA) sequentially on both sides from coarse to smooth grit starting from 600 grit with final polishing done at 1,200 grit (CarbiMet $^{\mathrm{TM}}$ Silicon Carbide grinding papers, Buehler, Lake Bluff, IL, USA) to achieve a consistent smooth and glossy flat surface.

This study used extracted natural carious teeth with dentin lesions. Consequently, large variability in terms of tooth and lesion size, consistency of lesions and distribution of lesions was expected. On the carious surface of the sliced samples, the size of the lesion in terms of pulpal depth and width was measured using a stereomicroscope (Olympus SZX16, Olympus Life Science, Tokyo, Japan). The pulpal depth was calculated from the dentinoenamel junction to the deepest point of the lesion, and the largest mesiodistal distance was considered as the width of the lesion. The point at which these dimensions coincide was taken as the approximate center of the lesion. This allowed for consistency in selecting the area of the lesion for assessment and enhanced reproducibility for future readings. Fluorescence, tactile and microhardness assessments were done at the center of the lesion on the carious surface and similarly on the corresponding sound dentin surface on each sample.

The 90 sliced samples, numbered from 1 to 90 , were randomly distributed using a computer-generated random number sequence into three groups ( $n=30$ per group) based on treatment with SDF. This randomization was done to minimize potential allocation bias and ensure that irrespective of the lesion size or dimensions, each sample had an equal chance of being assigned to any group. The SDF treatment protocol for each group was as follows-

Group A-No SDF (Control Group)

Group B-38\% SDF (Advantage Arrest ${ }^{\mathrm{TM}}$, Elevate Oral Care ${ }^{\circledR}$ LLC, FL, USA)

Group C-SDF with saturated Potassium Iodide (Riva Star, SDI, Victoria, Australia)

SDF application was done on the samples in Group B and $\mathrm{C}$ following the manufacturer's instructions on both surfaces (carious and sound). All samples were subjected to an additional rinsing with deionized water for $10 \mathrm{~s}$ and stored in an airtight container wrapped with gauze soaked in deionized water, a neutral storage solution that has been identified to produce minimal impact on dentin hardness (24) and maintain 100\% humidity. The samples in the humid airtight container were stored in an incubator Precision $^{\mathrm{TM}}$, Thermo Fisher Scientific, USA) set at $37^{\circ} \mathrm{C}$ for 1 week. After 1 week, microhardness, fluorescence and tactile assessments were repeated on the same surfaces. The study design is depicted as a flowchart in Figure 2 .

\section{Fluorescence Assessment}

In a dimly lit environment, the sample was illuminated using a handheld probe (SIROInspect ${ }^{\circledR}$, Sirona, Bensheim, Germany) with violet light of approximately $405 \mathrm{~nm}$ wavelength and observed through an orange filter. The fluorescence was recorded as red, pink or no fluorescence for each surface depending on the most predominant type noted. Fluorescence was assessed over the lesion on carious dentin surface and on the corresponding sound dentin surface of all samples before and 1 week after SDF application.

\section{Tactile Assessment}

Using a blunt-ended WHO probe, the tactile sensation was assessed on carious and sound dentin with care not to damage the lesion or the surface. Three points spanning the carious lesion and corresponding sound dentin surface were assessed. Recordings were noted as soft, leathery or firm. The most frequently occurring recording was considered as the tactile sensation for that surface. Tactile assessment was done for all samples before and 1 week after SDF application.

Both fluorescence and tactile assessments were done on a random subset of 30 samples independently by two calibrated blinded evaluators (MP and AFZ) for inter-examiner reliability and reassessed after 1 week by a single blinded evaluator (MP) to check for intra-examiner reproducibility. Evaluator MP performed the intra-examiner reliability as they eventually scored all surfaces for the study.

\section{Microhardness Assessment}

The mounted sample was placed under the indenter of a Vickers Microhardness testing machine (MicroMet ${ }^{\circledR}$ 2104, Buehler, Lake Bluff, IL, USA) and subjected to a load of $25 \mathrm{gf}$ for $10 \mathrm{~s}$ at each test point. Three adjacent measurements in Vicker's number (HV) were taken approximately over the center of the lesion on the carious dentin and on the corresponding sound dentin surface. The distances between the indentations were maintained at 100 \pm 25 microns to prevent interaction with work-hardened regions. To ensure the indentation on a softer tissue such as carious dentin is measured accurately and reliably, all indentations were measured using the DiaMet ${ }^{\mathrm{TM}}$ hardness testing software and measurements were consistently taken for all samples under $40 \times$ magnification so that margins of the indent were clearly visible. The average of the three measurements was considered the microhardness for that surface. Vickers microhardness testing was performed on both surfaces of all samples before and 1 week after SDF application.

\section{Statistical Analysis}

This was a pilot study with a sample size of $n=30$ per group. The effect size in this study will inform the sample size of a larger follow-up study that will maintain $80 \%$ power alongside a Type I error rate of $5 \%$. Descriptive statistics (means, standard deviations, medians, and interquartile ranges) were calculated. Intra and inter-examiner reliability for fluorescence and tactile assessment were calculated using Cohen's weighted kappa estimates. A kappa score of 0.81 or higher was considered to be almost perfect agreement based on Landis 


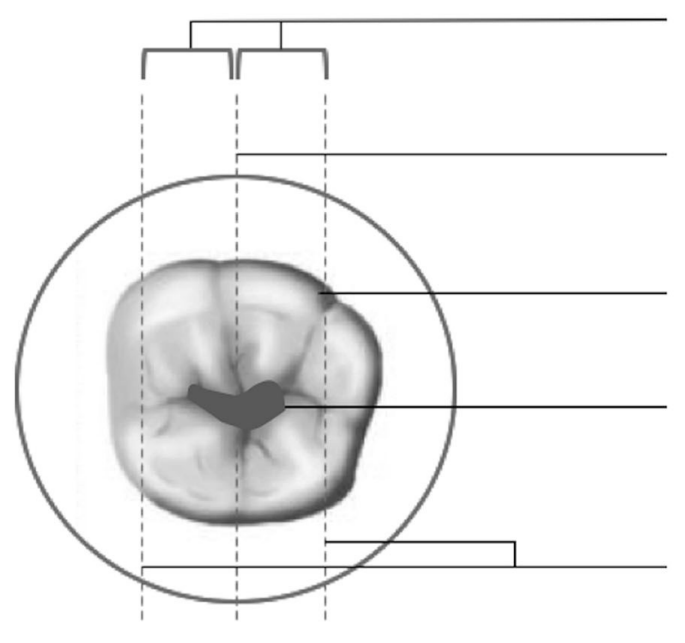

\section{Two longitudinal slices (samples) of the tooth exposing carious and sound dentin surfaces}

\section{Primary sectioning plane at the center of the carious lesion to expose carious dentin}

\section{Tooth mounted in acrylic resin}

Carious lesion on tooth surface (ICDAS 4-6)

\section{Secondary sectioning planes to expose sound dentin surface}

FIGURE 1 | Sectioning planes for sample preparation.

and Koch's guidelines (25). Spearman's rho was used to assess the correlation between fluorescence and tactile categories with microhardness values. For continuous variables, normality of data was assessed graphically as well as using the ShapiroWilk test and homogeneity of variances was assessed using Levene's test. Sphericity of data was assessed using Mauchly's test. Statistical significance for the difference between group means was assessed using Mixed ANOVA. $P$-values lower than 0.05 were considered statistically significant for the omnibus tests. Post-hoc comparisons were performed using Tukey's HSD. SAS Version 9.4 (SAS Inc., Cary, NC) was used for analysis.

\section{RESULTS}

Inter-examiner weighted kappa scores were 0.89 (95\% CI: 0.78-1.00) for concordance in assessing fluorescence and 0.88 (95\% CI: $0.78-0.99)$ for concordance in assessing the tactile sensation of carious lesions, indicating almost perfect agreement between the two calibrated examiners. Intra-examiner weighted kappa for examiner MP for scoring tactile sensation and fluorescence of carious lesions were both 0.96 (95\% CI: 0.89-1.00) indicating almost perfect reproducibility by the examiner in a 1-week follow-up exam.

A mixed ANOVA analysis demonstrated a statistically significant association between surface and $\triangle \mathrm{VMH}(p<0.0001)$, between group and $\triangle \mathrm{VMH}(p<0.0001)$ and finally between surface* group interaction and $\Delta \mathrm{VMH}(p=0.004)$. Within samples, the increase in microhardness of carious dentin surfaces was significantly larger than that of sound dentin surfaces $(p<0.0001)$. Between samples, a statistically significant increase in microhardness of both surfaces combined was seen in group $\mathrm{B}$ on receiving Advantage Arrest ${ }^{\mathrm{TM}}$ (Mean $\triangle \mathrm{VMH}: 19.99 \mathrm{HV} ; 95 \% \mathrm{CI}: 17.39-22.58)$ and Group $\mathrm{C}$ on receiving Riva Star (Mean $\triangle \mathrm{VMH}$ : $18.38 \mathrm{HV}$; 95\% CI: 15.7920.98), but Group A (control) which did not receive any SDF treatment showed no significant change in microhardness (Mean $\triangle \mathrm{VMH}$ : $-4.017 \mathrm{HV}$; 95\% CI: -6.61 to -1.42 ). The average change in microhardness $(\Delta \mathrm{VMH})$ on both surfaces for all groups after the assigned SDF protocol is shown in Figure 3.

When comparing the groups, carious and sound surfaces were analyzed separately and together. The $\triangle \mathrm{VMH}$ for experimental groups $\mathrm{B}$ and $\mathrm{C}$ were statistically significantly higher than the control group A (Group B vs. Group A: $p<0.0001$; Group C vs. Group A: $p<0.0001)$ for both carious and sound surfaces as well as when they were combined. This means that groups receiving SDF saw an increase in microhardness compared to the control group that was not treated with SDF, which did not see any increase in microhardness.

When carious and sound surfaces were combined, there was no statistically significant difference between the $\triangle \mathrm{VMH}$ of experimental groups B and C receiving SDFs (Group B vs. Group C: $p=0.661)$, seemingly indicating that Advantage Arrest ${ }^{\mathrm{TM}}$ and Riva Star increased microhardness to a similar extent. However, when carious and sound surfaces were separately analyzed, Group B receiving Advantage Arrest ${ }^{\mathrm{TM}}$ showed a statistically significantly larger $\triangle \mathrm{VMH}$ increase than Group $C$ receiving Riva Star on sound surfaces $(p=0.026)$. In carious surfaces, there was no statistically significant difference in $\triangle \mathrm{VMH}$ between Group $\mathrm{B}$ and Group $\mathrm{C}(p=0.146)$. Moreover, it was noted that there was no statistically significant association between type of surface and $\triangle \mathrm{VMH}$ in Group B $(p=0.809)$, meaning that microhardness of sound and carious surfaces increased to a similar extent with Advantage Arrest $^{\mathrm{TM}}$. Whereas, for SDF/KI, sound surfaces showed a statistically significantly lower $\triangle \mathrm{VMH}$ than carious surfaces $(p<0.0001)$, indicating that KI may have had a negative impact 


\section{Sample Selection Criteria (N=90)}

Posterior teeth

Coronal caries (ICDAS 4-6)

\section{Mounting and Sectioning}

Longitudinal slices

\section{Pre-SDF Assessments}

Fluorescence

(Red / Pink / No

Fluorescence)
Tactile sensation

(Soft / Leathery / Firm)
Vicker's

Microhardness test

(HV)

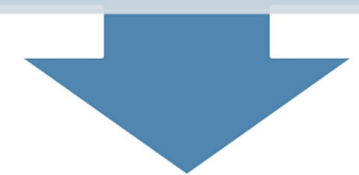

\section{Grouping and SDF Application ( $n=30)$}

\section{Group A - No SDF Group B - 38\% SDF Group C - SDF + KI \\ (Control) \\ (Advantage Arrest ${ }^{\mathrm{TM}}$, Elevate Oral Care, USA) \\ (Riva Star, SDI, Australia)}

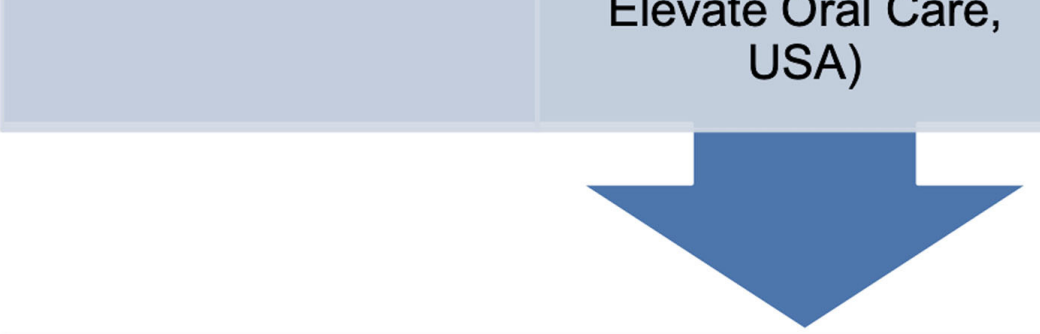

\section{Post-SDF Assessments (after 1 week storage)}

Vicker's

Microhardness test
Fluorescence (SIROInspect ${ }^{\circledR}$, Sirona, Germany)
Tactile Sensation 


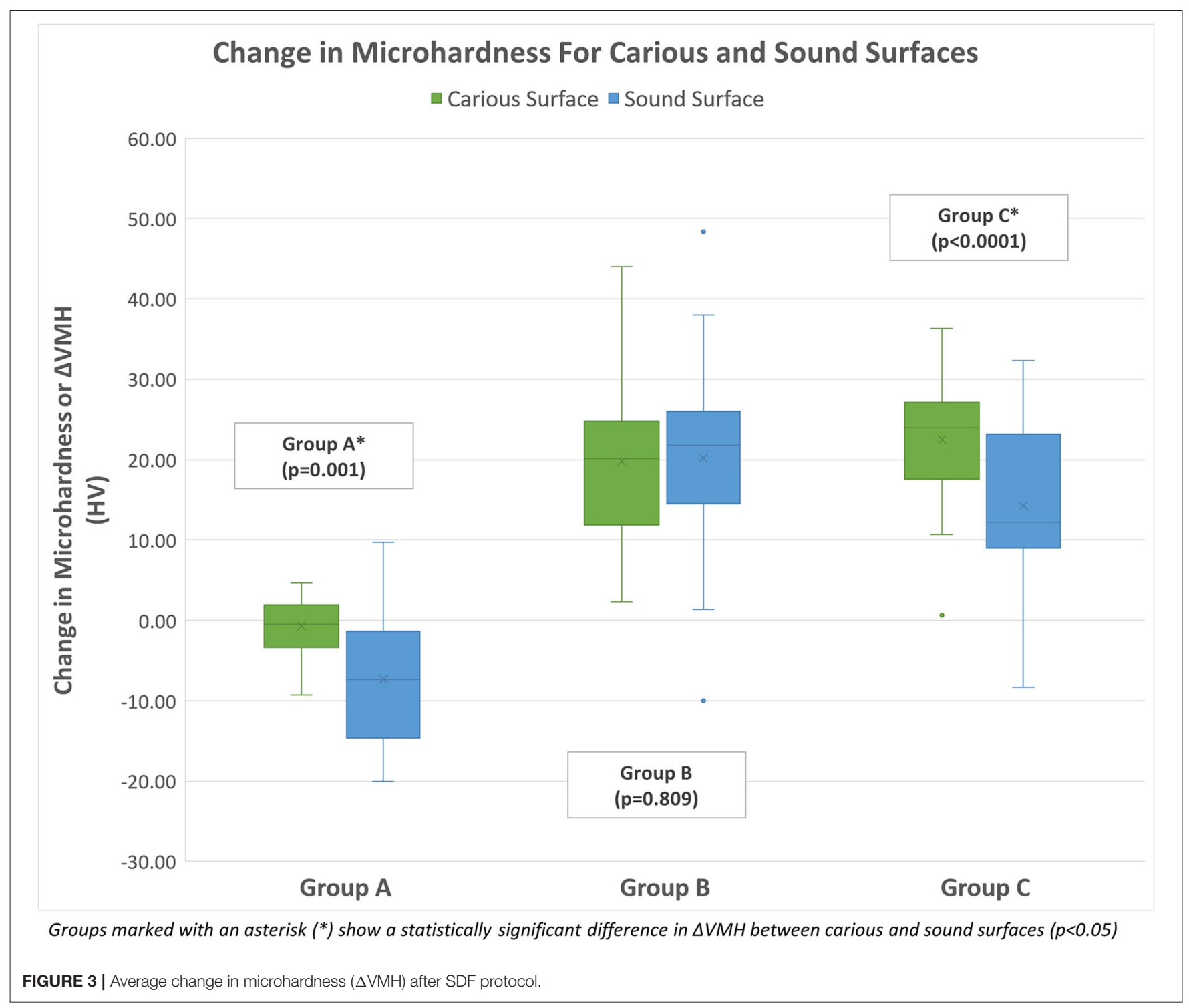

on the increase in microhardness of sound surfaces. $\triangle \mathrm{VMH}$ comparisons between all groups and surfaces are shown in Table 1.

It was found that Group $\mathrm{C}$ showed the smallest microhardness difference of $6.5 \mathrm{HV}(p=0.0002)$ between treated carious surfaces and untreated sound surfaces followed by Group B which showed a difference of $9.49 \mathrm{HV}(p<0.0001)$ and Group A receiving no SDF showed the largest difference of 26.62 HV ( $p<0.0001)$. It can be interpreted that Riva Star increased the microhardness of the carious surfaces closest to that of the corresponding sound surface of the tooth followed by Advantage Arrest ${ }^{\mathrm{TM}}$, which showed a larger difference between the surfaces.

The frequencies and percentages for tactile and fluorescence assessments for carious surfaces taken before and after SDF application are shown in Tables 2, 3, respectively. Sound surfaces in all groups showed no change in fluorescence or tactile readings. For carious surfaces overall, Spearman's rank correlation showed a statistically significant negative correlation between change in microhardness $(\triangle \mathrm{VMH})$ and change in fluorescence $(\Delta \mathrm{F})\left(\mathrm{r}_{\mathrm{s}}=-0.269, p=0.01\right)$, and between change in microhardness $(\Delta \mathrm{VMH})$ and change in tactile sensation $(\Delta \mathrm{T})$ $\left(\mathrm{r}_{\mathrm{s}}=-0.588, p<0.001\right)$. Therefore, an increased hardness can be correlated with a firmer tactile assessment. There was a statistically significant small positive correlation between change in fluorescence $(\Delta \mathrm{F})$ and change in tactile sensation $(\Delta T)$ for carious surfaces $\left(r_{s}=0.226, p=0.032\right)$. Spearman's rank correlation was also calculated after stratifying by group. The stratified correlations were very weak, ranging from $r_{s}=0.149$ between $\triangle \mathrm{VMH}$ and $\Delta \mathrm{T}$ in Group $\mathrm{A}$ to $\mathrm{r}_{\mathrm{s}}=0$ between $\Delta \mathrm{T}$ and $\Delta \mathrm{F}$ in Group $\mathrm{A}$. None of the stratified correlation estimates were statistically significant $(p>0.05)$. Fluorescence and Vickers microhardness indentations of a sample in each group before and after SDF protocol are shown in Figure 4. 
TABLE 1 | Change in microhardness [Mean (Std. Dev.)] based on type of surface assessed and type of SDF treatment.

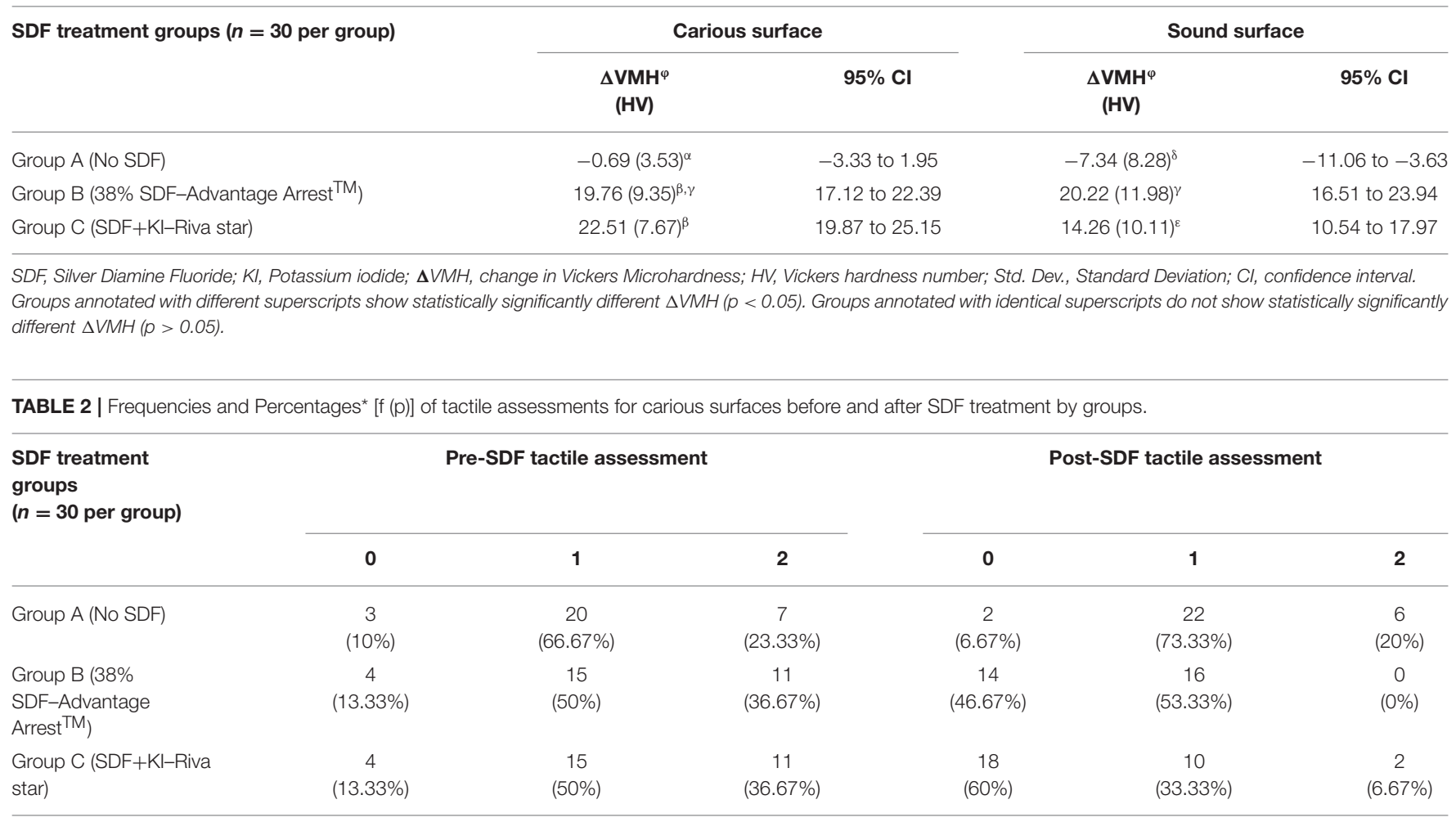

Tactile, 0 = firm, 1 = leathery, 2 = soft.

${ }^{*}$ Percentage, $p=(f / n)^{*} 100$.

SDF, silver diamine fluoride; KI, potassium iodide; $f$, frequency; $p$, percentage; Pre-SDF, before SDF treatment; Post-SDF, after SDF treatment.

TABLE 3 | Frequencies and Percentages ${ }^{\star}[\mathrm{f}(\mathrm{p})]$ of fluorescence assessments for carious surfaces before and after SDF treatment by group.

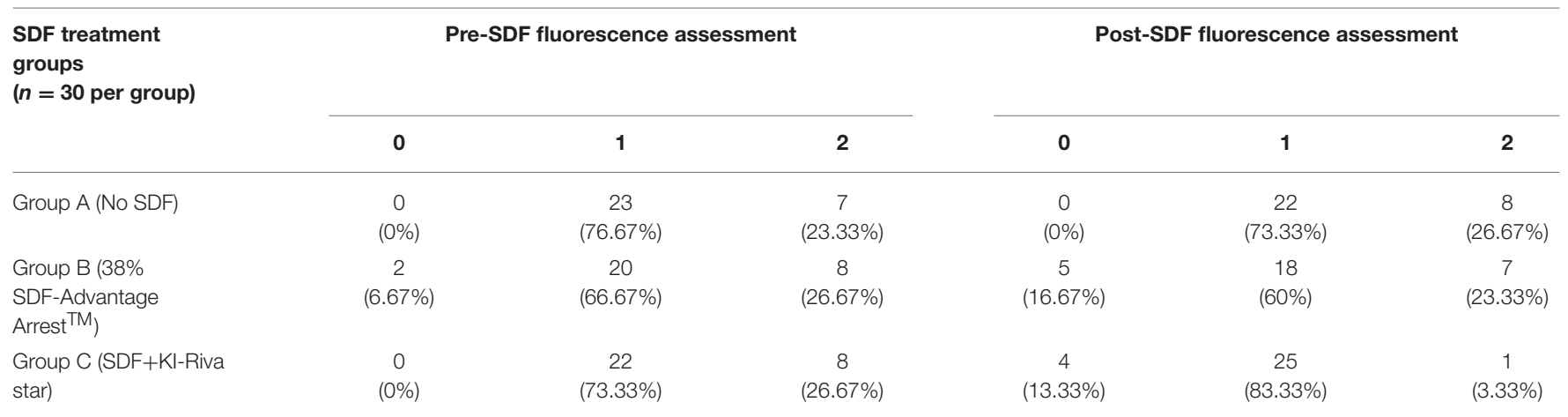

Fluorescence, $0=$ no fluorescence, 1 = pink fluorescence, 2 = red fluorescence.

*Percentage, $p=(f / n)^{\star} 100$.

SDF, silver diamine fluoride; KI, potassium iodide; $f$, frequency; $p$, percentage; Pre-SDF, before SDF treatment; Post-SDF, after SDF treatment.

\section{DISCUSSION}

This study investigated the effect of SDF on microhardness of carious and sound dentin in permanent teeth. To the authors' knowledge, this is the first study to assess this effect in permanent posterior teeth with natural coronal lesions. Chu's clinical trials on primary teeth reported microhardness to be higher in the outermost dentinal surface of arrested carious lesions receiving regular application of $38 \% \mathrm{SDF}$ for 30 months compared to active carious lesions (26). Results of the present study were in general agreement with the outcomes of the prior study (26) evaluating the microhardness of dentin with $38 \%$ SDF treatment. However, given that the prior study was not conducted on permanent teeth, it was not possible to directly establish comparisons as measuring hardness on primary teeth has very different clinical implications. Furthermore, the prior study differs from the current study in that it was a clinical trial where the teeth remained in the oral cavity post-SDF treatment till extraction. In-vivo factors such 


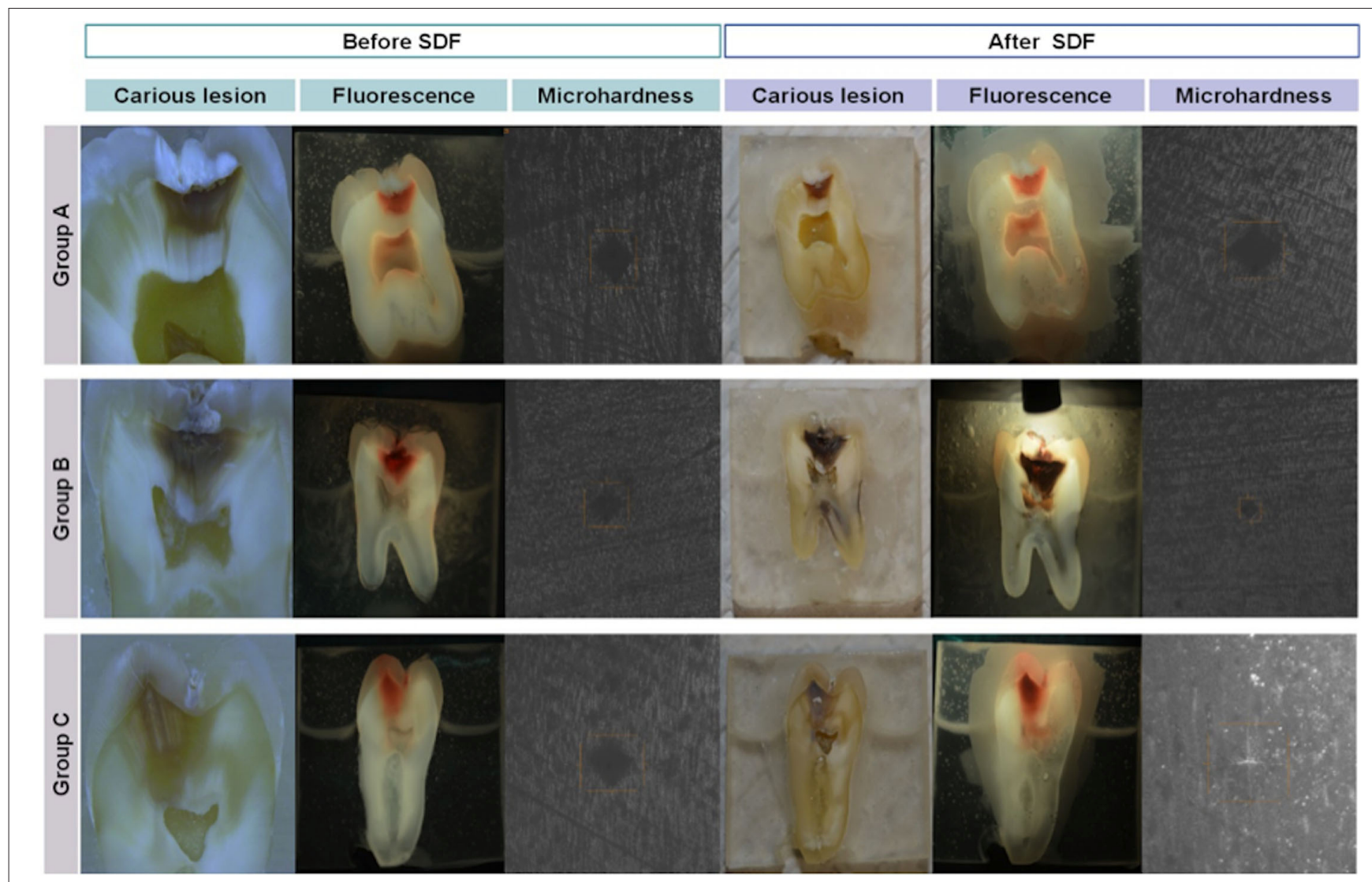

FIGURE 4 | Carious lesions, fluorescence, and microhardness indents recorded before and after SDF in all groups.

as the remineralizing effect of saliva, pulpal reparative dentin formation or calcified tissue apposition within dentin may serve as a source of differences that could potentially influence the effect of SDF. Additionally, Chu's trial did not evaluate the effect of silver diamine fluoride with potassium iodide (KI) on dentin microhardness.

A saturated KI solution has been known to reduce the staining induced on the tooth by SDF (16). Therefore, in addition to $38 \%$ SDF (Advantage Arrest ${ }^{\mathrm{TM}}$ ), the effects of a readily available combination of SDF+KI (Riva Star) were also investigated in the present study. It was revealed that both preparations did not significantly differ from each other in improving microhardness when used on carious surfaces. However, Advantage Arrest ${ }^{\mathrm{TM}}$ improved microhardness of sound surfaces more than Riva Star, suggesting that KI had a negative impact on the microhardness increase on a sound dentin surface.

Horst stated that some SDF-treated lesions continued to progress, especially those in the inner third of the dentin (15). Chu's trials concluded that remineralization with fluoride helps to recover dentin hardness in primary teeth only to a certain depth, beyond which it is insignificant (26). The UCSF protocol for caries arrest using SDF states that silver and fluoride ions may penetrate $\sim 25$ microns into enamel and 50-200 microns into dentin (15). Furthermore, a study using SEM revealed that silver ions from SDF cover the surface of sound dentin and may further penetrate up to 20 microns into dentinal tubules, occluding them (27). This penetration potential could explain the current finding of an increase in the microhardness of sound dentin after SDF treatment. The authors acknowledge that the present study's scope was limited to assessing the surface effect of SDF and did not extend to measuring the depth of penetration or the depth of the effect on carious permanent teeth which can be considered a limitation of the study. The depth of effect on microhardness will be of great significance in determining the maximum depth of the lesion at which SDF could be effective; a topic of interest for further research.

Given SDF's propensity to stain the tooth, it is commonly used in posterior teeth in conjunction with class I, class II, and certain class $\mathrm{V}$ restorations where esthetics may not be of prime importance. The study utilized samples that were sliced longitudinally to expose carious lesions on one side of the sample and a sound surface on the other. With this form of slicing, the microhardness measurements were assessed on what would clinically be an axial wall of a preparation and not the pulpal wall, both differing in the direction in which dentinal tubules are cut (pulpal walls see transversely cut dentinal tubules whereas axial walls see longitudinally cut tubules). Previous studies (28) reported the hardness values of sound dentin to range from 50 
to $70 \mathrm{KNH}$ with variations depending on the location and the mineralization of intertubular dentin that may be influenced by the orientation of dentinal tubules in that region. While the hardness values in the present study were comparable to the range reported, they may not be suitable to accurately judge class I or class II scenarios, which can also be considered as a limitation of the study. Nevertheless, this study model allowed for optimizing standardization for comparison between sound and carious surfaces wherein each tooth served as its own control.

At the time of the study, there were no established clinical protocols or guidelines for the application of SDF. The authors adopted the SDF protocol followed by Tufts University clinics wherein a lesion is typically revisited 1 week after SDF application to ensure clinical hardening before attempting a restoration. Therefore, samples in the study were assessed for fluorescence, tactile sensation, and microhardness 1 week after SDF application to replicate clinical practice. It has been reported the SDF's activity decreases over time and repeated applications may reinforce the effect (29). Horst and colleagues recommended a biannual application up to 24 months after an initial application at the diagnostic visit (15). However, if a patient warranting restorative treatment is unable to return for a follow-up visit or in cases of long wait times involved in hospital dentistry, silver-modified atraumatic restorations or SMART restorations are often relied upon wherein a restorative material such as conventional glass ionomer is immediately placed following SDF application at the initial visit (30). The results of the present study, having evaluated only a single application of SDF, are applicable to silver modified atraumatic restorative techniques (SMART restorations) or when the intention is to restore the cavitated lesion after a single application and must be considered with caution while extrapolating for long-term outcomes of conventional restorative treatment.

Visible red fluorescence has been suggested to be a good indicator for the presence and activity of bacteria on dentin (31). This concept is adopted in the fluorescenceaided caries excavation technology (SIROInspect ${ }^{\circledR}$, Sirona, Bensheim, Germany) which allows the operator to clinically detect fluorescence emitted by bacterial metabolites and remove bacterially-infected dentin in carious lesions more successfully than by visual-tactile methods (32). Interestingly, this study saw samples with a positive indication of carious activity by fluorescence and visible appearance yet having relatively higher hardness values and hard tactile sensations, implying despite what appears a heavily florescent bacterial load, there was little effect on the dentin structure in these samples. In such cases, SDF may render an added advantage of arresting bacterial metabolic activity thus prevent the weakening of dentin structure caused by acid metabolites, thereby implicating its use on red/pink fluorescing firm lesions without the need for removing dentin structure to achieve caries arrest and dentin preservation. A study that looked at the dentin bacterial microbiome found that one-third of the tactile assessments of hard dentin still displayed some fluorescence, either pink or red, concluding that dentin layers displayed diverse bacterial $\operatorname{taxa}(33)$.
Staining materials on tooth surfaces can negatively affect fluorescence-aided caries excavation procedures and their results. In this study, lesions treated with Advantage Arrest ${ }^{\mathrm{TM}}$ were expected to show obscurement of fluorescence whereas Riva Star was not expected to impact fluorescence due to KI's stain reducing potential. However, the evident staining considerably obscured the fluorescence in both the groups, suggesting that KI did not completely eliminate the staining. In light of this, a limitation of this study was that no objective assessment could be performed due to lack of set criteria other than a subjective visual recording to measure fluorescence on stained surfaces. In the future, it would be valuable to identify an objective assessment, such as assessing fluorescence by image analyses. In the current study, it was interesting to note that despite a large obscurement of fluorescence in the Advantage Arrest ${ }^{\mathrm{TM}}$ group, mild specks were still visible on the lesions 1 week after SDF application. The authors speculated that this finding could be the result of heavy contamination with increased bacterial load rendering the antimicrobial nature of silver ineffective or that the additional 10-s rinse after SDF application removed the residual antimicrobial silver ions from the surface. A compounded effect of both heavy contamination and rinsing of SDF could imply that the specks of fluorescence were a result of both an increased bacterial load and a reduced amount of silver ions on the surface.

Traditionally, microhardness testing is done in a laboratory involving equipment that cannot be utilized in vivo. A study using a novel portable hardness testing machine introduced by Shimizu called Cariotester (SUK-971, Sanei ME Corporation, Yokohama, Japan; Cariotester) found microhardness of active carious lesions to correlate with the fluorescence detected using DIAGNOdent (Kavo, Biberach, Germany) (34, 35). However, more studies are required to evaluate the usefulness of this novel tool. Most microhardness studies continue to implement the traditional laboratory-based systems.

In order to associate these laboratory-based microhardness tests to clinical parameters, this study secondarily looked for any correlations between microhardness and chair side assessments such as fluorescence and tactile sensations. The present study found a low correlation between an increase in dentin microhardness and a categorical reduction in fluorescence using FACE technology, but a stronger correlation between increase in microhardness and a categorical increase in firmness of tactile sensation. However, when stratified by group, the correlations estimates were not statistically significant. The present study, though appearing inconsistent with Iwami's findings (35), cannot be directly compared with Iwami's study due to differences in tests and methodology. For instance, Iwami conducted Knoop hardness tests through a portable chair side testing system but the present study utilized a laboratory-based Vickers hardness test. To measure fluorescence, Iwami used DIAGNOdent but the current study implemented the FACE technology. A study by Trippe using FACE technology found that tactile assessments of soft, leathery, and hard corresponded with red fluorescence, pink fluorescence, and no fluorescence, respectively (33). However, the present study found only a 
weak correlation between fluorescence and tactile sensation of carious dentin and therefore was not in agreement with Trippe's findings.

The overall picture indicates that it may be possible for a clinician to judge the change of microhardness to a considerable extent with a chair side tactile assessment, but this is not likely with fluorescence. However, the results of this study are limited to in-vitro situations; further investigation is required to substantiate or reject these findings clinically.

\section{CONCLUSIONS}

Within the limitations of this study, it was concluded that microhardness of SDF treated dentin surfaces increased as compared to non-SDF treated surfaces after a single application. Riva Star (SDF+KI) improved carious dentin microhardness closer to that of a sound dentin surface than Advantage Arrest $^{\mathrm{TM}}$ (38\% SDF). The addition of KI affected the change in microhardness negatively for sound surfaces. Overall, an increase in microhardness correlated with a categorically firmer tactile sensation but not with fluorescence. Further research is needed to establish these correlations clinically and make a recommendation for clinical benefit.

\section{DATA AVAILABILITY STATEMENT}

The raw data supporting the conclusions of this article will be made available by the authors, without undue reservation.

\section{REFERENCES}

1. Ericson D, Kidd E, McComb D, Mjör I, Noack MJ. Minimally Invasive Dentistry - concepts and techniques in cariology. Oral Health Prev Dent. (2003) 1:59-72.

2. Mertz-Fairhurst EJ, Curtis JW Jr, Ergle JW, Rueggeberg FA, Adair SM. Ultraconservative and cariostatic sealed restorations: results at year 10. J Am Dent Assoc. (1998) 129:55-66. doi: 10.14219/jada.archive.1998.0022

3. Schwendicke F, Dörfer CE, Paris S. Incomplete caries removal: a systematic review and meta-analysis. J Dent Res. (2013) 92:306-14. doi: 10.1177/0022034513477425

4. Qvist V. Longevity of restorations: the "death spiral". In Fejerskov O, Kidd EAM, editors. Dental Caries: The Disease and Its Clinical Management. Oxford: Blackwell Munksgaard (2008). p. 444-55.

5. Oong EM, Griffin SO, Kohn WG, Gooch BF, Caufield PW. The effect of dental sealants on bacteria levels in caries lesions: a review of the evidence. J Am Dent Assoc. (2008) 139:271-358. doi: 10.14219/jada.archive.2008.0156

6. Schwendicke F, Stolpe M, Meyer-Lueckel H, Paris S, Dörfer CE. Costeffectiveness of one- and two-step incomplete and complete excavations. $J$ Dent Res. (2013) 92:880-7. doi: 10.1177/0022034513500792

7. Schwendicke F, Paris S, Stolpe M. Cost-effectiveness of caries excavations in different risk groups - a micro-simulation study. BMC Oral Health. (2014) 14:153. doi: 10.1186/1472-6831-14-153

8. Barros MMAF, De Queiroz Rodrigues MI, Muniz FWMG, Rodrigues LKA. Selective, stepwise, or nonselective removal of carious tissue: which technique offers lower risk for the treatment of dental caries in permanent teeth? A systematic review and meta-analysis. Clin Oral Investig. (2020) 24:52132. doi: 10.1007/s00784-019-03114-5

9. Splieth $\mathrm{CH}$, Banerjee A, Bottenberg P, Breschi L, Campus G, Ekstrand KR, et al. How to intervene in the caries process in children: a joint ORCA

\section{ETHICS STATEMENT}

The studies involving human participants were reviewed and approved by Social, Behavioral \& Educational Research IRBTufts University. Written informed consent for participation was not required for this study in accordance with the national legislation and the institutional requirements.

\section{AUTHOR CONTRIBUTIONS}

MP and AZ conducted the study over a period of 6 months at Tufts University School of Dental Medicine and drafted this research paper. YK substantially contributed to the study design and methodology development. SJ worked on the statistical analysis of the data and drafted the analysis segment of the paper. All authors finalized and approved the contents of the paper.

\section{FUNDING}

This study was funded by the Department of Comprehensive Care Research Funds at Tufts University School of Dental Medicine, Boston MA.

\section{ACKNOWLEDGMENTS}

The authors would like to sincerely thank the Gavel Laboratory at Tufts University School of Dental Medicine, Boston for providing access to the required machines and materials to perform the sample preparation and microhardness testing. and EFCD expert Delphi Consensus Statement. Caries Res. (2020) 54:297305. doi: $10.1159 / 000507692$

10. Oen KT, Thompson VP, Vena D, Caufield PW, Curro F, Dasanayake A, et al. Attitudes and expectations of treating deep caries: a PEARL Network survey. Gen Dent. (2007) 55:197-203.

11. Weber CM, Alves LS, Maltz M. Treatment decisions for deep carious lesions in the public health service in Southern Brazil. J Public Health Dent. (2011) 71:265-70. doi: 10.1111/j.1752-7325.2011.00258.x

12. Schwendicke F, Meyer-Lueckel H, Dörfer C, Paris S. Attitudes and behaviour regarding deep dentin caries removal: a survey among German dentists. Caries Res. (2013) 47:566-73. doi: 10.1159/000351662

13. Burgess JO, Vaghela PM. Silver diamine fluoride: a successful anticarious solution with limits. Adv Dent Res. (2018) 29:1314. doi: $10.1177 / 0022034517740123$

14. Zhao IS, Gao SS, Hiraishi N, Burrow MF, Duangthip D, Mei ML, et al Mechanisms of silver diamine fluoride on arresting caries: a literature review. Int Dent J. (2018) 68:67-76. doi: 10.1111/idj.12320

15. Horst JA, Ellenikiotis H, Milgrom PL. UCSF protocol for caries arrest using silver diamine fluoride: rationale, indications and consent. $J$ Calif Dent Assoc. (2016) 4 4:16-28.

16. Patel J, Anthonappa RP, King NM. Evaluation of the staining potential of silver diamine fluoride: in vitro. Int J Paediatr Dent. (2018) 28:112. doi: 10.1111/ipd.12401

17. Knight GM, McIntyre JM, Mulyani. The effect of silver fluoride and potassium iodide on the bond strength of auto cure glass ionomer cement to dentine. Aust Dent J. (2006) 51:42-5. doi: 10.1111/j.1834-7819.2006.tb00399.x

18. González-Cabezas C. The chemistry of caries: remineralization and demineralization events with direct clinical relevance. Dent Clin North Am. (2010) 54:469-78. doi: 10.1016/j.cden.2010.03.004 
19. Angker L, Nockolds C, Swain MV, Kilpatrick N. Correlating the mechanical properties to the mineral content of carious dentine-a comparative study using an ultra-micro indentation system (UMIS) and SEM-BSE signals. Arch Oral Biol. (2004) 49:369-78. doi: 10.1016/j.archoralbio.2003.12.005

20. Chu CH, Lo EC, Lin HC. Effectiveness of silver diamine fluoride and sodium fluoride varnish in arresting dentine caries in Chinese pre-school children. $J$ Dent Res. (2002) 81:767-70. doi: 10.1177/0810767

21. Llodra JC, Rodriguez A, Ferrer B, Menardia V, Ramos T. Morato M. Efficacy of silver diamine fluoride for caries reduction in primary teeth and first permanent molars of schoolchildren: 36-month clinical trial. J Dent Res. (2005) 84:721-4. doi: 10.1177/154405910508400807

22. Zhi QH, Lo EC, Lin HC. Randomized clinical trial on effectiveness of silver diamine fluoride and glass ionomer in arresting dentine caries in preschool children. J Dent. (2012) 40:962-7. doi: 10.1016/j.jdent.2012.08.002

23. Slayton RL, Urquhart $\mathrm{O}$, Araujo MWB, Fontana M, GuzmánArmstrong S, Nascimento MM, et al. Evidence-based clinical practice guideline on nonrestorative treatments for carious lesions: a report from the American Dental Association. J Am Dent Assoc. (2018) 149:837-49.e19. doi: 10.1016/j.adaj.2018.07.002

24. Aydin B, Pamir T, Baltaci A, Orman MN, Turk T. Effect of storage solutions on microhardness of crown enamel and dentin. Eur J Dent. (2015) 9:2626. doi: 10.4103/1305-7456.156848

25. Landis RJ, Koch GG. The measurement of observer agreement for categorical data. Biometrics. (1977) 33:159-74. doi: 10.2307/2529310

26. Chu $\mathrm{CH}$, Lo EC. Microhardness of dentine in primary teeth after topical fluoride applications. J Dent. (2008) 36:38791. doi: 10.1016/j.jdent.2008.02.013

27. Willershausen I, Schulte D, Azaripour A, Weyer V, Briseno B, Willershausen B. Penetration potential of a silver diamine fluoride solution on dentin surfaces. An ex vivo study. Clin Lab. (2015) 61:1695-701. doi: 10.7754/Clin.Lab.2015.150401

28. Kinney JH, Marshall SJ, Marshall GW. The mechanical properties of human dentin: a critical review and re-evaluation of the dental literature. Crit Rev Oral Biol Med. (2003) 14:13-29. doi: 10.1177/154411130301400103

29. Yee R, Holmgren C, Mulder J, Lama D, Walker D, van Palenstein Helderman W. Efficacy of silver diamine fluoride for Arresting Caries Treatment. J Dent Res. (2009) 88:644-7. doi: 10.1177/0022034509338671

30. Alvear Fa B, Jew JA, Wong A, Young D. Silver Modified Atraumatic Restorative Technique (SMART): an alternative caries prevention tool. Stoma Edu J. (2016) 3:18-24. doi: 10.25241/2016.3(2).15
31. Lennon AM, Buchalla W, Brune L, Zimmermann O, Gross U, Attin T. The ability of selected oral microorganisms to emit red fluorescence. Caries Res. (2006) 40:2-5. doi: 10.1159/0000 88898

32. Lennon AM. Fluorescence-aided caries excavation (FACE) compared to conventional method. Oper Dent. (2003) 2 8:341-5.

33. Trippe LH, Ribeiro AA, Azcarate-Peril MA, Preisser JS, Wang R, Zandona AF. Is fluorescence technology a promising tool for detecting infected dentin in deep carious lesions? Caries Res. (2020) 54:205-17. doi: 10.1159/0005 05643

34. Shimizu A, Nakashima S, Nikaido T, Sugawara T, Yamamoto T, Momoi Y. Newly developed hardness testing system, "Cariotester": measurement principles and development of a program for measuring Knoop hardness of carious dentin. Dent Mater J. (2013) 32:643-7. doi: 10.4012/dmj.2012-312

35. Iwami Y, Yamamoto H, Hayashi M. Validity of a portable microhardness testing system (Cariotester) for diagnosis of progression in active caries lesions. Dent Mater J. (2013) 32:667-72. doi: 10.4012/dmj.2012-328

Conflict of Interest: AZ is a consultant for Colgate-Palmolive, Calcivis, GreenMark, and Dental Quest.

The remaining authors declare that the research was conducted in the absence of any commercial or financial relationships that could be construed as a potential conflict of interest.

Publisher's Note: All claims expressed in this article are solely those of the authors and do not necessarily represent those of their affiliated organizations, or those of the publisher, the editors and the reviewers. Any product that may be evaluated in this article, or claim that may be made by its manufacturer, is not guaranteed or endorsed by the publisher.

Copyright (C) 2021 Prakash, Kang, Jain and Zandona. This is an open-access article distributed under the terms of the Creative Commons Attribution License (CC BY). The use, distribution or reproduction in other forums is permitted, provided the original author(s) and the copyright owner(s) are credited and that the original publication in this journal is cited, in accordance with accepted academic practice. No use, distribution or reproduction is permitted which does not comply with these terms. 